\section{IN THE NEWS}

\section{Chromosomes 'worn} down' by stress

It is not only our spirits that are eroded by constant anxiety

and worry; it seems that our chromosomes are also,

literally, worn down by stress.

This is the conclusion of a

study published in the

Proceedings of the National Academy of Sciences that

asked whether the

prematurely aged appearance

of emotionally stressed

people was reflected in the

genome of their cells.

Elissa Epel and colleagues used telomere length which declines in older people - to compare the biological age of the blood cells of mothers that were caring for a sick child with that of mothers with a healthy child. They found a striking correlation between the duration of care giving and the accelerated shortening of telomeres. Mothers looking after a chronically ill child are "...subjected to the kind of grinding day-to-day stress that can wear anyone down", sympathized Elizabeth Blackburn, one of the authors on the study "... and the longer their stress continued, the more it appeared they aged biologically" (San Francisco Chronicle, 30 November 2004). However, even those mothers with healthy children who felt emotionally stressed had shorter telomeres. And the blood cells in the highstress group had aged by a staggering 9-17 years. "How we perceive the world can matter more than our objective reality", says Elissa Epel (New Scientist, 29 November 2004) and she points out that this research is "... a dramatic example of the mind-body link" (San Francisco Chronicle, 30 November 2004).

But how do our emotions penetrate to the heart of our cells? "Right now," says Dr Blackburn, "that is the black box, and that's what we're going to study next." (The New York Times, 30 November, 2004.

Shannon Amoils

\section{LIPID METABOLISM}

\section{A two-step affair}

Reporting in Science, Rudolf Zechner and colleagues describe the identification of a novel enzyme, adipose triglyceride lipase (ATGL), which catalyses the hydrolysis of triglycerides to diglycerides in mammalian adipose tissue. The discovery of this once elusive second lipase is an important step forward in the fight against obesity-related disorders.

Adipocytes, which make up the bulk of adipose tissue, have a typical morphology that is characterized by the presence of a large lipid droplet - a spherical organelle that contains abundant triglycerides. During periods of starvation, this repository of fat is mobilized by the catabolism of triglycerides to non-esterified fatty acids, which enter the circulation and provide cells with a source of energy. Until recently, researchers believed that hormone-sensitive lipase (HSL) was the sole rate-determining lipolytic enzyme in adipose tissue, but the phenotype of HSL-knockout mice changed this widely held view. These animals were not obese, and had accumulated diglycerides in adipocytes, which indicated that, even in the absence of HSL, asyet-unidentified lipases efficiently degraded triglycerides to diglycerides.

Zechner and co-workers set out to find these enzymes by carrying out homology searches for murine and human proteins that were structurally similar to known lipases. One putative candidate, ATGL, was identified and, as both human and mouse adipose tissue express high levels of $A T G L$ mRNA, it seemed as if this protein could well be the 'missing lipase’.

Zechner and his team expressed ATGL in a mammalian non-adipocyte cell line, and compared the in vitro activity and substrate specificity of ATGL with that of transfected HSL. Both proteins were enzymatically active but, whereas HSL showed a broad substrate specificity that included triglycerides, cholesterol esters and retinyl esters, ATGL activity was only directed towards triglycerides. Also, ATGL predominantly hydrolysed the first ester bond of triglyceride - which resulted in the conversion of triglycerides to diglycerides. Western-blot analysis and immunofluorescence microscopy confirmed that, in mouse adipocytes, adenovirus-encoded ATGL associated with the lipid droplet - the expected cellular location of a lipolytic enzyme. Furthermore, adipocytes that were infected with the adenoviral ATGL construct released larger amounts of glycerol and fatty acids compared with control cells. And the overexpression of ATGL caused an increase in diglyceride levels, which indicates that the enzyme has a physiological role in the catabolism of triglycerides.

In view of these results, the authors predict that lipids are mobilized in a two-step process: ATGL

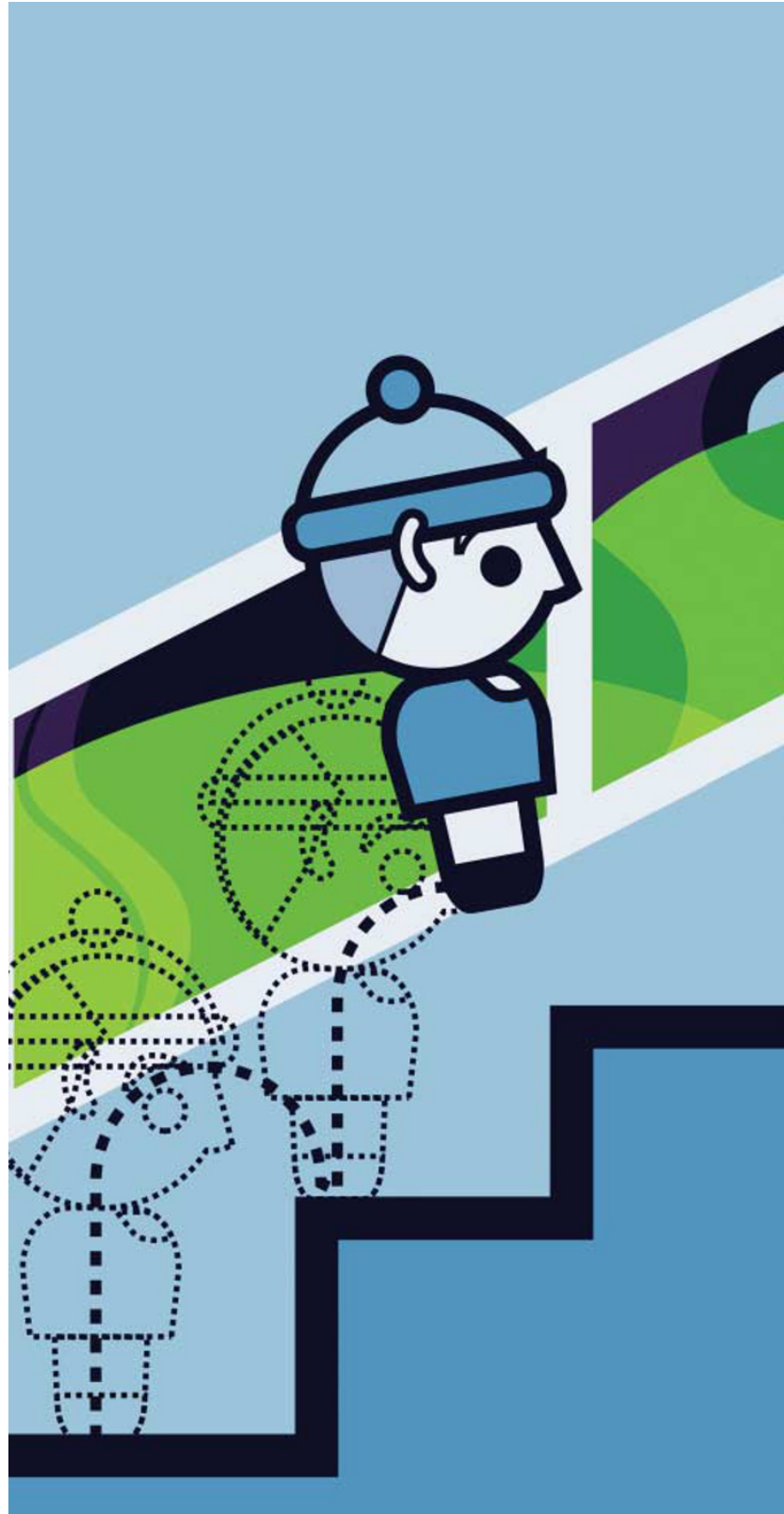

hydrolyses the first ester bond of triglycerides, which results in the formation of diglycerides that can then be broken down by HSL. As dysfunctional fatty-acid mobilization contributes to the metabolic defects that characterize type- 2 diabetes, the addition of ATGL to the mammalian lipolytic repertoire might provide researchers with a novel target for the treatment of this disorder.

Shannon Amoils

\section{(4) References and links}

ORIGINAL RESEARCH PAPER Zimmerman, R. et al. Fat mobilization in adipose tissue is promoted by adipose triglyceride lipase. Science $\mathbf{3 0 6}$, 1383-1386 (2004)

FURTHER READING Villena, J. A. et al. Desnutrin, an adipocyte gene encoding a novel patatin domain-containing protein, is induced by fasting and glucocorticoids: ectopic expression of desnutrin increases triglyceride hydrolysis. J. Biol. Chem. 279, 47066-47075 (2004) | Jenkins, C. M. et al. Identification, cloning, expression, and purification of three novel human calcium-independent phospholipase A2 family members possessing triacylglycerol lipase and acylglycerol transacylase activities J. Biol. Chem. 279, 48968-48975 (2004) 